\title{
Nota CienTífica \\ Dieta del pato quetru no volador Tachyeres pteneres en un humedal marino de Chiloé, sur de Chile
}

\author{
Diet of the Magellanic Flightless Steamer Duck Tachyeres pteneres
} in a marine wetland of Chiloé, southern Chile

\author{
Rodrigo Araneda ${ }^{1}$, Claudio N. Tobar', Jaime R. Rau ${ }^{1,5}$ \\ y Jaime A. Cursach ${ }^{1,3,4}$
}

\begin{abstract}
'Laboratorio de Ecología, Departamento de Ciencias Biológicas \& Biodiversidad, Universidad de Los Lagos, Casilla 933, Campus Osorno, Osorno, Chile. *jrau@ulagos.cl

${ }^{2}$ Departamento de Ciencias Básicas, Universidad Santo Tomás, Los Carrera 753, Osorno, Chile

${ }^{3}$ Programa de Doctorado en Ciencias, Escuela de Postgrado, Universidad de Los Lagos, Campus Chinquihue, Puerto Montt, Chile ${ }^{4}$ Laboratorio de Investigación Socioambiental, Programa ATLAS, Departamento de Ciencias Sociales, Universidad de Los Lagos, Casilla 933, Campus Osorno, Osorno, Chile
\end{abstract}

\begin{abstract}
The Magellanic Flightless Steamer Duck (Tachyeres pteneres) is the only marine anatid founded along the coastline of Chile and Argentina, from Valdivia to Tierra del Fuego. There is little published information about the quantitative diet of this species. Previously, a single study of its breeding diet was reported for Guapiquilán Island, Chiloé, southern Chile. We studied T. pteneres' winter diet during 2011 and 2012 in Bahía Caulín, Chiloé, southern Chile. We found that statistically Cancridae crabs (100 and $100 \%$, respectively) and Majidae (96.0-71.4\%, respectively) were the most common items in the diet of this species.
\end{abstract}

Key words: Food habits, marine ducks, southern Chile, crabs

\section{INTRODUCCIÓN}

El pato quetru no volador Tachyeres pteneres (Forster, 1844) es el representante más grande del género Tachyeres (Goodall et al. 1951, Tobar et al. 2011) y el único anátido marino presente en las costas de Chile (Schlatter \& Simeone 1999). Se le considera un ave marino-costera debido a su alta dependencia trófica y permanencia en el medio marino (Schlatter \& Simeone 1999, Cursach \& Rau 2009). Es un excelente buceador (Livezey \& Humphrey 1985), y una de las aves marinas más características que se distribuye desde la Isla de Chiloé hasta el Cabo de Hornos en el lado del Pacífico de América del Sur y las islas de Tierra del Fuego y de los Estados por el lado del Atlántico (Livezey \& Humphrey 1985, Jaramillo 2005, McGehee \& Eitniear 2007). En Chile, es endémica de la zona subantártica y habita la costa del sur del país, distribuyéndose sólo desde Valdivia $\left(39^{\circ} \mathrm{S}\right)$ hasta Tierra del Fuego $\left(54^{\circ} \mathrm{S}\right)$ (Jaramillo 2005). A nivel internacional es una especie catalogada como de menor preocupación, debido a que su población se considera estable, sin entregarse mayores antecedentes (IUCN 2017).

La información ecológica publicada para patos quetrus no voladores detalla su distribución local y describe algunos sitios de nidificación (Bernath 1965, Cursach \& Rau 2009), conducta de forrajeo (Ryan et al. 1988), sus depredadores (Straneck et al. 1983, Rozzi \& Sherriffs 2003), problemas de conservación (Schüttler et al. 2009) y observaciones sobre el comportamiento reproductivo (Livezey \& Humphrey 1984,
Livezey \& Humphrey 1985, Medina 1989). En relación a su dieta existen antecedentes descriptivos publicados (Delacour \& Mayr 1949, Goodall et al. 1951, Jehl 1973). Sin embargo, Livezey (1989) realizó previamente en Ushuaia, Argentina, un estudio cuantitativo en base al análisis de los contenidos estomacales $(n=20)$ de esta especie. De acuerdo a los antecedentes recopilados en Chile sólo existe un estudio cuantitativo previo el cual fue realizado en la temporada reproductiva del verano de 2009 en isla Guapiquilán, Chiloé (Tobar et al. 2011). Lo anterior establece una oportunidad para incrementar el conocimiento al respecto, por lo que el objetivo de este estudio fue determinar y cuantificar las categorías de presas que forman parte de la dieta invernal de T. pteneres, en un humedal marino de Chiloé, sur de Chile.

\section{Materiales Y MÉTODOS}

Durante junio 2011 y julio 2012 se colectaron 25 y 35 muestras fecales $(\mathrm{N}=60)$, respectivamente, de pato quetru no volador entre las coordenadas $41^{\circ} 48^{\prime} \mathrm{S} ; 7^{\circ} 37^{\prime} \mathrm{O}$ en el humedal marino de Bahía Caulín. De acuerdo con Encabo et al. (2012) y Vilugrón et al. (2016), esta localidad de 176 ha está ubicada en la costa sur del canal Chacao, porción noreste de la Isla Grande de Chiloé, Región de Los Lagos, sur de Chile (Fig. 1). Es denominado localmente 'Santuario de las Aves de Caulín' y fue declarado como Área de Importancia para la Conservación de Aves (i.e., sitio AICA) por BirdLife Internacional (Soazo et 
al. 2009). Desde la línea de marea baja a la línea de marea alta, la distancia es ca., de $600 \mathrm{~m}$ en marea de sicigia. El intermareal está sujeto a la constante extracción de productos del mar, especialmente la recolección y cultivo del alga 'pelillo' (Gracilaria chilensis) y ostra chilena (Ostrea chilensis). Las colectas de fecas fueron realizadas durante horarios de mañana ( $n=3$ días de muestreos consecutivos en ambos inviernos), en los sitios utilizados por el pato quetru como áreas de descanso (Fig. 1). Cada feca corresponde a una deposición por individuo adulto, identificado con ayuda de un telescopio terrestre Barska $^{\odot}(20-60$ X 60 mm), mediante las características de su coloración y plumaje. Aunque las fecas antiguas fueron removidas después de su colecta inicial, no pueden asociarse a individuos discretos ya que desconocemos su tasa de defecación promedio diaria. Una vez observados defecar los animales se procedió a colectar las fecas en el lugar donde estos se encontraban. Las muestras fueron puestas en una estufa Memmert $^{\circledR}$, secadas durante $24 \mathrm{~h}$ a $60^{\circ} \mathrm{C}$ y procesadas en laboratorio de manera estándar. Los restos no digeridos fueron examinados bajo lupa estereoscópica Wild M3 Heerbrugg (10
X 23), utilizándose como nivel de resolución taxonómica sólo hasta la categoría de Familia o Clase, debido a la alta degradación de los restos de presas contenidos en las fecas. Para su identificación se utilizaron las partes duras de los invertebrados (e.g., quelas/exoesqueletos de crustáceos, conchas de moluscos), guías taxonómicas y claves dicotómicas (Rathbun 1937, Zagal \& Hermosilla 2001, Aldea \& Valdovinos 2005) y colecciones de invertebrados de los propios autores. Una vez clasificadas las muestras se procedió a determinar la frecuencia absoluta de ocurrencia para cada Familia o Clase en función del número total de fecas con restos analizadas. Para comparar las frecuencias absolutas de las categorías tróficas (i.e., Familias y Clases), intra e interanualmente, en las dietas no reproductivas invernales de esta especie se utilizó la prueba de $\chi^{2}$, prueba de bondad de ajuste y tabla de contingencia, respectivamente. Las frecuencias observadas fueron agrupadas para satisfacer el criterio de que las frecuencias esperadas fuesen también iguales o mayores a cinco. Para ello se utilizó el paquete computacional estadístico en línea VassarStats: Website for Statistical Computation $\left({ }^{\circledR}\right.$ Richard Lowry 1998-2017) ${ }^{1}$.
Figura 1. Ubicación geográfica de los sitios donde se colectaron las fecas de Tachyeres pteneres presentes en Bahía Caulín, Isla Grande de Chiloé, sur de Chile / Geographic location of the sites where the feces of Tachyeres pteneres were collected present in Bahía Caulín, Isla Grande de Chiloé, southern Chile

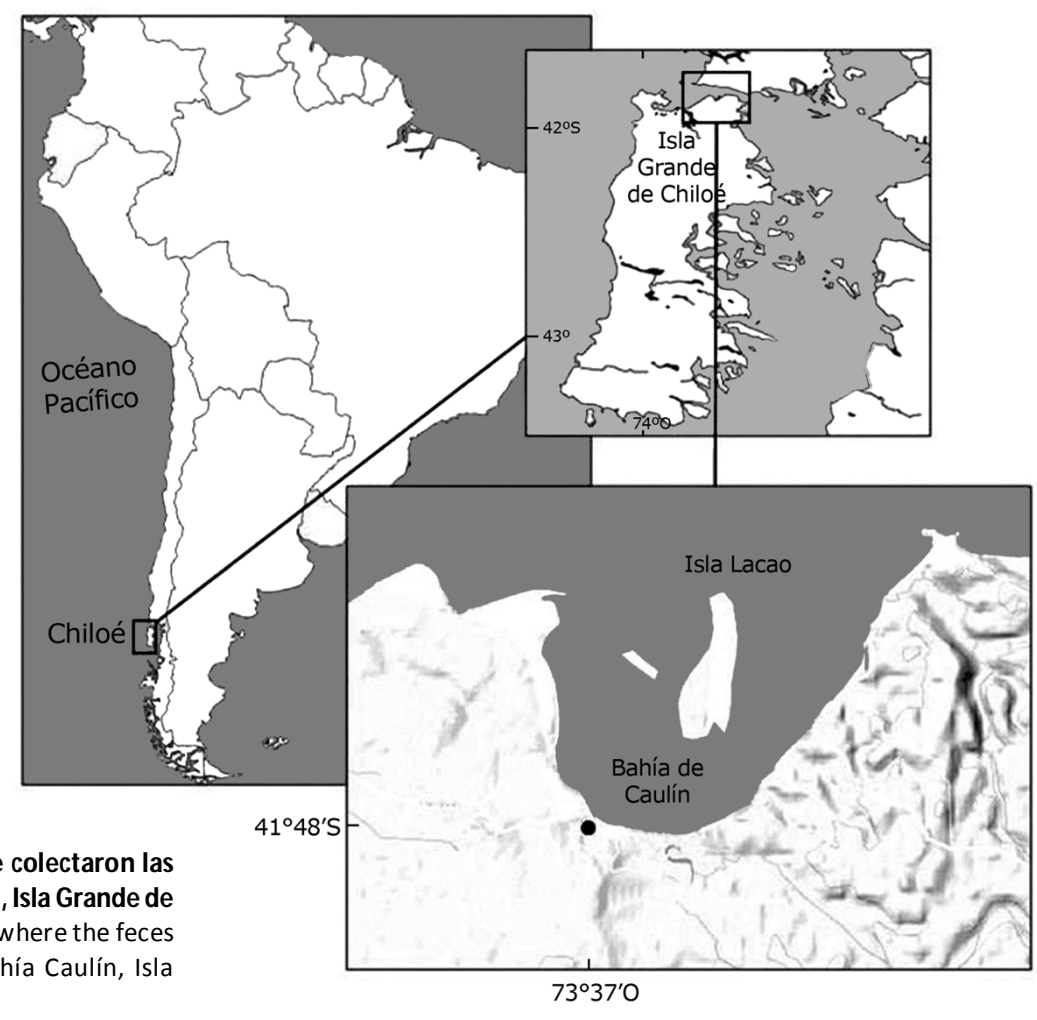

${ }^{1}<$ http://www.vassarstats.net/> 


\section{RESULTADOS Y DISCUSIÓN}

En las 60 fecas analizadas se encontraron categorías tróficas correspondientes a 7 Familias y 3 Clases taxonómicas (Tabla 1). Las frecuencias relativas más altas correspondieron a representantes de las Familias Cancridae (100 y 100\%, respectivamente, $\mathrm{n}=25)$ y Majidae (96,0 y 71,4\%, respectivamente, $\mathrm{n}=35$ ) (Tabla 1 ). El análisis estadístico de los resultados para el invierno 2011 muestra la importancia significativa en la dieta de sólo dos categorías tróficas (Cancridae y Majidae $)\left(\chi^{2}=9,58 ;\right.$ g.l. $\left.=2 ; P=0,008\right)$, mientras que para el invierno 2012 el predominio de tres categorías tróficas (Cancridae, Majidae y otras categorías), que no fue significativo $\left(\chi^{2}=3,15 ;\right.$ g.l. $\left.=2 ; P=0,207\right)$. El número de categorías tróficas fluctuó entre un rango de 1 a 6 (invierno 2011) y 1 a 8 (invierno 2012) Familias o Clases por feca. Al comparar las frecuencias absolutas observadas de las diferentes categorías tróficas en las 60 fecas, entre las temporadas no reproductivas correspondientes a los inviernos de 2011 y 2012, se encontraron diferencias estadísticas significativas $\left(\chi^{2}=11.67 ;\right.$ g.l. $=2 ; P=$ $0,003)$. Los antecedentes cualitativos y cuantitativos de la dieta de los patos quetrus no voladores señalan el consumo de diversos invertebrados marinos, potencialmente ciertas especies de peces y algas submareales (Goodall et al. 1951, Jehl 1973, Livezey 1989, Tobar et al. 2011). Al igual que en el estudio de Tobar et al. (2011), no se encontraron gastrolitos. En las fecas analizadas en este trabajo se identificaron restos duros de invertebrados marinos y restos de microalgas, probablemente consumidas de manera oportunista. En observaciones de terreno en la zona de estudio y para ambos inviernos se registraron avistamiento de estos patos alimentándose en la cercanía de bosques de huiros (Macrocystis pyrifera), de manera similar a lo informado por Medina (1989) en bahía Yaldad, Chiloé ( $\left.43^{\circ} 07^{\prime} \mathrm{S} ; 7^{\circ} 37^{\prime} \mathrm{O}\right)$, en donde la mayor parte de su alimento se obtuvo entre los bosques de esta macroalga, basando su dieta principalmente en crustáceos y moluscos. Esta especie puede albergar y dar protección a una gran diversidad de organismos, incluyendo otras especies de algas, peces y moluscos, entre otras, y la presencia de huiros (i.e., especie 'clave') es crucial para mantener la diversidad y organización de las comunidades ecológicas (e.g., Plana et al. 2007). En cuanto al consumo de categorías tróficas, existió un mayor consumo de crustáceos malacostracos. El consumo de crustáceos también ha sido observado en la costa de Calbuco

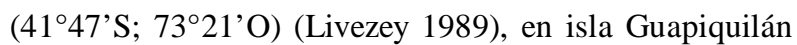

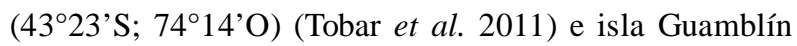

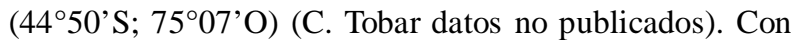
respecto a la clase Insecta, se encontraron pupas probablemente pertenecientes al orden Diptera, y también probablemente consumidas de manera oportunista. Tal vez su presencia esté
Tabla 1. Identidad taxonómica de las Familias o Clases de invertebrados marinos, frecuencia absolutasy relativas (\%) en fecas de patos quetrus no voladores durante la temporada no reproductiva invernal de 2011 y 2012 en Bahía Caulín, Chiloé, sur de Chile / Taxonomic identity of Families or Classes of marine invertebrates absolute and relative frequencies (\%) in feces of Magellanic Flightless Steamer-ducks during the winter non-breeding season of 2011-2012 in Bahía Caulín, Chiloé, southern Chile

\begin{tabular}{lcc}
\hline No de Familias o Clases & $\begin{array}{c}\text { Frecuencias } \\
\text { absolutas y } \\
\text { relativas (\%) }\end{array}$ & $\begin{array}{c}\text { Frecuencias } \\
\text { absolutas y } \\
\text { relativas (\%) }\end{array}$ \\
\hline Cancridae & $25(100 \%)$ & $35(100 \%)$ \\
Majidae & $24(96,0 \%)$ & $25(71,4 \%)$ \\
Ostreidae & $0(0,0 \%)$ & $7(20,0 \%)$ \\
Phaeophycedae & $0(0,0 \%)$ & $12(34,4 \%)$ \\
Insecta & $0(0,0 \%)$ & $16(45,7 \%)$ \\
Mytilidae & $4(16,0 \%)$ & $2(5,7 \%)$ \\
Veneridae & $0(0,0 \%)$ & $1(2,9 \%)$ \\
Bivalvia no determinados & $2(8,0 \%)$ & $0(0.0 \%)$ \\
Trochidae & $1(4,0 \%)$ & $1(2,9 \%)$ \\
Gastropoda & $1(4,0 \%)$ & $0(0,0 \%)$ \\
Total de fecas & 25 & 35 \\
\hline
\end{tabular}

asociada al consumo por parte de los patos de fecas y algas en descomposición y puede ser considerado como incidental. Los resultados obtenidos en este trabajo indican que las presas consumidas, preferentemente por individuos adultos dequetrus, son predominantemente crustáceos, y en menor frecuencia, moluscos bentónicos. Pese a su estado de conservación, según la IUCN (2017), se debe aumentar el esfuerzo de estudio sobre la ecología trófica de este anátido marino, cuyas poblaciones en estado silvestre se encontrarían actualmente amenazadas por especies silvestres introducidas y domésticas (i.e., Neovison vison, Canis lupus familiaris, respectivamente, Liljesthröm et al. 2014) y la continua y recurrente alteración de los ambientes costeros del sur de Chile (e.g., pérdida de vegetación costera y actividades intensivas de acuicultura).

\section{Agradecimientos}

A los Núcleos de Investigación BIODES y BIODES 2.0 y a la Dirección de Investigación \& Postgrado de la Universidad de Los Lagos por financiar los costos de esta publicación. También a Christopher B. Anderson y Soraya Sade por la revisión del Abstract y la edición del texto, respectivamente. 


\section{LiTERATURA CITADA}

Aldea C \& C Valdovinos. 2005. Moluscos del intermareal rocoso del centro-sur de Chile $\left(36^{\circ}-38^{\circ} \mathrm{S}\right)$ : taxonomía y clave de identificación. Gayana 69: 364-396.

Bernath E. 1965. Observations in southern Chile in the southern hemisphere autumn. Auk 82:95-101.

Cursach J \& J Rau. 2009. Abundancia y nidificación del pato quetru no volador Tachyeres pteneres en Bahía Lenca, Seno de Reloncaví, sur de Chile. Boletín del Museo Nacional de Historia Natural, Chile 58:97-100.

Delacour J \& E Mayr. 1949. La familia Anatidae. Hornero, Argentina 9: 24-79.

Encabo M, J Rau-Acuña, V Vasquez, C Tobar, DP Barreto \& J Cursach. 2012. Aviturismo en conservación: experiencias en Argentina y Chile. Serie Turismo y Conservación, 50 pp. Editorial de la Universidad Nacional del Comahue, Neuquén.

Goodall JJ, AW Johnson \& RA Philippi. 1951. Las aves de Chile, su conocimiento y sus costumbres, $445 \mathrm{pp}$. Platt Establecimientos Gráficos, Buenos Aires.

IUCN. 2017. Red list of threatened species. <www.iucnredlist .org>

Jaramillo A. 2005. Aves de Chile, 240 pp. Ediciones Lynx, Barcelona.

Jehl JR. 1973. The distribution of marine birds in Chilean waters in winter. Auk 90: 114-135.

Liljesthröm M, L Fasola, A Valenzuela, A Raya-Rey \& A Schiavini. 2014. Nest predators of Flightless Steamer-Ducks (Tachyeres pteneres) and Flying Steamer-Ducks (Tachyeres patachonicus). Waterbirds 37: 210-214.

Livezey B. 1989. Feeding morphology, foraging behavior, and foods of steamer-ducks (Anatidae: Tachyeres). Occasional Papers Museum of Natural History, The University of Kansas 126: $1-41$.

Livezey B \& P Humphrey. 1984. Diving behaviour of steamerducks Tachyeres spp. Ibis 126: 257-260.

Livezey B \& P Humphrey. 1985. Territoriality and interspecific aggression in steamer-ducks. Condor 87: 154-157.

McGehee S \& J Eitniear. 2007. Kleptoparasitism of Magellanic Flightless Steamer-ducks (Tachyeres pteneres) by Kelp gulls (Larus dominicanus. Boletín SAO (Sociedad Antioqueña de Ornitología) 17: 141-144.

Medina G. 1989. Contribución a la ecología del pato quetru no volador (Tachyeres pteneres) y la relación de esta especie con la mitilicultura en Yaldad, Chiloé insular. Tesis de Medicina Veterinaria, Universidad Austral de Chile, Valdivia, 87 pp.
Plana J, A Mancilla, M Palacios \& P Navarro. 2007. Estudio poblacional de Macrocystis pyrifera (L.) C. Agardh (Laminariales: Phaeophyta) en ambientes protegido y expuesto al oleaje en Tierra del Fuego. Gayana 71: 66-75.

Rathbun M. 1937. The oxystomatous and allied crabs of America, Smithsonian Institution United States National Museum. Bulletin 166: 1-370.

Rozzi R \& M Sherriffs. 2003. El visón (Mustela vison Schreber, Carnivora: Mustelidae), un nuevo mamífero exótico para la isla Navarino. Anales del Instituto de la Patagonia 31:97-104.

Ryan PG, AL Bosman \& PAR Jockey. 1988. Notes on the foraging behaviour of Magellanic Flightless Steamer Ducks and Flying Steamer Ducks. Wildlfowl 39: 29-33.

Schlatter R \& A Simeone. 1999. Estado del conocimiento y conservación de las aves en mares chilenos. Estudios Oceanológicos 18: 25-33.

Schüttler E, R Klenke, S Mcgehee, R Rozzi \& K Jax. 2009. Vulnerability of ground-nesting waterbirds to predation by invasive American mink in the Cape Horn Biosphere Reserve, Chile. Biological Conservation 142: 1450-1460.

Soazo PO, I Rodríguez-Jorquera, P Arrey-Garrido \& A Jaramillo. 2009. Chile. En: Devenish C, DF DíazFernández, RP Clay, I Davidson \& I Yépez-Zabala (eds). Important Bird Areas Americas - Priority sites for biodiversity conservation. Quito, BirdLife International, Quito. BirdLife Conservation Series 16: 125-134.

Straneck R, B Livezey \& P Humphrey. 1983. Predation on steamer-ducks by killer whale. Condor 85: 255-256.

Tobar C, A Arriagada, J Rau, J Cursach, C Suazo \& R Márquez. 2011. Dieta del pato quetru no volador Tachyeres pteneres en isla Guapiquilán, Chiloé, sur de Chile. Boletín Chileno de Ornitología 17: 103-108.

Vilugrón-Torres J, J Rau-Acuña \& ME Encabo. 2016. Comportamiento de aves y visitantes: humedal de Caulín, Isla Grande de Chiloé, Chile, 56 pp. Serie Turismo y Conservación, Editorial de la Universidad Nacional del Comahue, Neuquén.

Zagal C \& C Hermosilla. 2001. Guía de invertebrados marinos del litoral valdiviano, 217 pp. Universidad Austral de Chile, Valdivia. 